\title{
Alveolar process changes associated with administration of nucleos(t)ide analogue (sofosbuvir) in rat model
}

\section{Zmiany wyrostka zębodołowego związane z zastosowaniem analogu nukleozydów (sofosbuwiru) w badaniu u szczurów}

\author{
Dina Badawy Elsayed Farag A-D,F, Dalia Abdel-Hameed El-Baz ${ }^{\mathrm{A}, \mathrm{C}, \mathrm{D}, \mathrm{F}}$, Zeinab Amin Salem ${ }^{\mathrm{A}, \mathrm{B}, \mathrm{E}, \mathrm{F}}$ \\ Department of Oral Biology, Faculty of Dentistry, Cairo University, Egypt \\ A - research concept and design; $B$ - collection and/or assembly of data; $C$ - data analysis and interpretation; \\ $D$ - writing the article; $E$ - critical revision of the article; $F$ - final approval of the article
}

Address for correspondence

Zeinab A. Salem

E-mail: abodarwish2005@yahoo.com

Funding sources

None declared

Conflict of interest

None declared

Received on 0ctober 14, 2017

Reviewed on November 17, 2017

Accepted on December 27, 2017

DOI

$10.17219 / \mathrm{dmp} / 81592$

Copyright

○ 2018 by Wroclaw Medical University

and Polish Dental Society

This is an article distributed under the terms of the

Creative Commons Attribution Non-Commercial License

(http://creativecommons.org/licenses/by-nc-nd/4.0/)

\begin{abstract}
Background. Sofosbuvir is a nucleotide compound that has proved to be among the most potent orally available antiviral treatments. Infrequent but serious adverse events have been reported with the use of oral nucleos(t)ide analogues.

Objectives. Investigating sofosbuvir-induced alterations in the rat mandibular alveolar process.

Material and methods. In the study, 30 adult male albino rats were used and divided randomly into following groups: group 1, group 2 and group 3 (10 rats per group). Group 1 served as a control, group 2 received sofosbuvir through oral gavage at a dose of $40 \mathrm{mg} / \mathrm{kg} / \mathrm{day}$ for 6 weeks, and group 3 was similar to group 2 but received sofosbuvir for 12 weeks. The animals were sacrificed at the end of the experiment. The mandibles were dissected and examined histologically as well as by scanning electron microscope (SEM) and energy dispersive $X$-ray unit (EDX).

Results. Histologically, group 1 showed normal alveolar process. In group 2, histopathological changes occurred as bone trabeculae demonstrated obvious Howship's lacuna of osteoclasts. In group 3, bone trabeculae exhibited multiple degenerated areas as well as apparent vacuolization. Scanning electron microscopic examination revealed smooth alveolar bone architecture in group 1. On the other hand, groups 2 and 3 demonstrated irregular bone architecture with formation of multiple pores. EDX analysis demonstrated the highest calcium concentration in the control group, while the lowest was found in group 3. Statistical anaIysis of the EDX results revealed a statistically significant difference among the studied groups as the p-value was $<0.01$
\end{abstract}

Conclusions. It has been concluded that sofosbuvir induced apparent alterations in the rats' alveolar bone. This effect was exaggerated in a longer period of drug administration. The sofosbuvir-induced alterations might be attributed mainly to mitochondrial toxicity. The effect had been clearly shown histologically and morphologically as well as in bone mineral (calcium) content.

Key words: hepatitis ( virus, alveolar bone, sofosbuvir, nucleos(t)ide analogues, scanning microscopy

Słowa kluczowe: wirus zapalenia wątroby typu C, kość wyrostka zębodołowego, sofosbuwir, analogi nukleozydów, mikroskopia skaningowa 


\section{Introduction}

Nucleos $(\mathrm{t})$ ide inhibitors are analogues of the naturally occurring polymerase substrates. They form the basis of antiviral therapy for a number of chronic viral diseases such as herpes simplex virus (HSV), human immunodeficiency virus (HIV), and hepatitis B virus (HBV) ${ }^{1,2}$ Nucleos(t)ides analogues resemble physiological nucleos(t)ides in terms of uptake and metabolism, and are incorporated into newly synthesized DNA. The incorporation of these compounds into DNA may induce either the termination of chain elongation or the accumulation of mutations in viral offspring. Some of these drugs also inhibit key enzymes involved in the generation of the purine and pyrimidine nucleotides and RNA synthesis, and directly activate caspase cascade. All of these effects may lead to cell death. ${ }^{3,4}$

Infrequent but serious adverse events have been reported with the use of oral nucleos(t)ide analogues. All nucleos(t)ide analogues have a "black box warning" regarding potential mitochondrial toxicity in their product labeling. The differences in analogue toxicity might result from a combination of cellular uptake, organellar transport, metabolic activation, incorporation and removal or degradation from the system. ${ }^{5}$

Sofosbuvir, acyclovir and tenofovir are nucleotide compounds proved to be among the most potent orally available antiviral treatments. These drugs exhibit high efficacy and a wide therapeutic index, with demonstrated utility in a number of chronic viral infections. ${ }^{6}$

Considering the published data regarding the adverse events associated with the use of oral nucleos(t)ide analogues, the present study was undertaken to evaluate the possible effects of sofosbuvir - a nucleo(s)tide analogue - on the mandibular alveolar process in albino rats through light microscopy, scanning electron microscopic examination and energy dispersive $\mathrm{X}$-ray analysis (EDX).

\section{Material and methods}

\section{Ethical statement}

All experiments were conducted in the animal house of the Faculty of Medicine, Cairo University in Egipt according to the recommendations and approval of the Ethics Committee on animal experimentation of the Faculty of Oral and Dental Medicine, Cairo University in Egipt.

\section{Experimental design}

A total of 30 rats were used in the current study, weighing approx. $200 \mathrm{~g}$. The animals were kept in a 12-hour light/dark cycle, at a temperature of $22^{\circ} \mathrm{C} \pm 2^{\circ} \mathrm{C}$ with relative humidity $50 \% \pm 20 \%$. The animals were fed on standard chow pellets and tap water ad libitum for the entire test period. The rats were randomly assigned into 3 groups of 10 rats each. Group 1 served as a control and the rats received distilled water through oral gavage. Group 2 received sofosbuvir (Gratisovir ${ }^{\circledR}$ tablets, Pharco Pharmaceuticals, Alexandria, Egypt) dissolved in distilled water $(8 \mathrm{mg} / \mathrm{mL})$ through oral gavage at a dose of $40 \mathrm{mg} / \mathrm{kg} /$ day for 6 weeks. ${ }^{7}$ Group 3 received sofosbuvir dissolved in distilled water $(8 \mathrm{mg} / \mathrm{mL})$ through oral gavage at a dose of $40 \mathrm{mg} / \mathrm{kg} /$ day for 12 weeks. ${ }^{7}$ All animals were sacrificed by ketamine overdose and the mandibles were dissected. The right side of each mandible was used for light microscopic examination, forming a total of 30 bone segments (10 specimens from each group). The other 30 bone segments ( 10 specimens from each group) of the left sides of the mandibles were used for scanning electron microscopic (SEM) examination as well as analysis of the constituent element (calcium concentration) using an energy dispersive X-ray unit attached to the SEM.

\section{Light microscopic examination}

All the specimens were fixed in $10 \%$ neutral formalin for $48 \mathrm{~h}$, washed, soaked in 10\% ethylene diamine tetraacetic acid (EDTA) for decalcification for 4 weeks, and then rinsed in distilled water. The specimens were dehydrated in ascending grades of alcohol and embedded in paraffin. Mesiodistal sectioning of the right side of the jaw was carried out. Histological sections were prepared of $5 \mu \mathrm{m}$ thickness. The sections were subjected to hematoxylin and eosin staining according to the conventional method. Histopathological examination was performed using light microscope.

\section{Scanning electron microscopic examination}

The specimens were fixed in $2.5 \%$ glutaraldehyde in phosphate buffer ( $\mathrm{pH}$ 7.4) for $6 \mathrm{~h}$. The specimens were then dehydrated in ascending grades of ethanol for $10 \mathrm{~min}$ at each passage and 20 min twice in absolute ethanol, subjected to critical point drying according to standard procedures for SEM processing, and left to dry in air at room temperature for 3 days. The specimens were then mounted on scanning electron microscope stubs and studied with a Quanta 250 Field Emission Gun (FEG) SEM (Thermo Fisher Scientific, Waltham, USA).

\section{Energy dispersive X-ray analysis}

The quantitative composition of calcium in the studied samples was determined by EDX spot measurement, EDX line scan and element mapping. The EDX analysis unit works as an integrated feature of the Quanta 250 FEG SEM. 


\section{Statistical analysis}

All the data obtained from the EDX analysis was statistically evaluated. This data was represented as mean values $\pm \mathrm{SD}$, which was then statistically compared among the 3 studied groups. One-way analysis of variance (ANOVA) test for more than 2 independent samples was used, followed by Tukey's post hoc test for pair wise comparison. The results were expressed in the form of $\mathrm{p}$-values that were considered significant when the probability value was $\leq 0.05$ and highly significant when the probability value was $\leq 0.01$. All statistical calculations were performed using following computer programs: Microsoft Excel 2007 and Statistical Package for the Social Science (SPSS) (SPSS Inc., IBM Corp., Armonk, USA) v. 15 for Microsoft Windows.

\section{Results}

\section{Histological results}

Group 1 (control group) showed normal alveolar bone architecture. The spongiosa consisted of interconnecting bone trabeculae containing osteocytes filling their lacunae. The bone trabeculae enclosed marrow cavities that were intensely lined with osteoblasts and filled with fibrocellular tissue (Fig. 1).

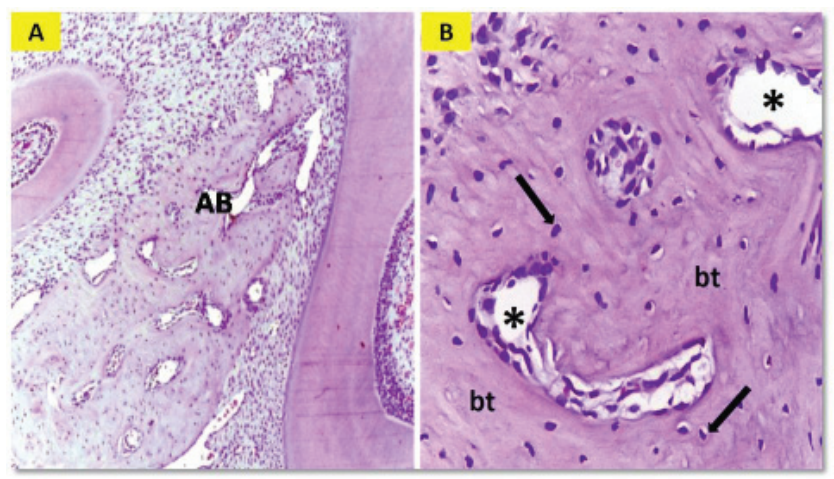

Fig. 1. Photomicrograph of group 1 (control): (A) showing normal interdental alveolar bone $(A B)$ consisting of interconnecting bone trabeculae (bt), osteocytes filling their lacunae (arrows) and marrow spaces (asterisks) (A: H\&E ×100, B: H\&E ×400)

Group 2 demonstrated bone trabeculae with randomly distributed osteocytes as well as scalloped reversal lines. Howship's lacuna of osteoclasts were also clearly observed (Fig. 2).

Group 3 exhibited bone trabeculae with multiple degenerated areas as well as apparent vacuolization. The osteocytes were irregularly arranged. Moreover, some osteocyte lacunae were seen empty. Multiple reversal lines were clearly demonstrated (Fig. 3).
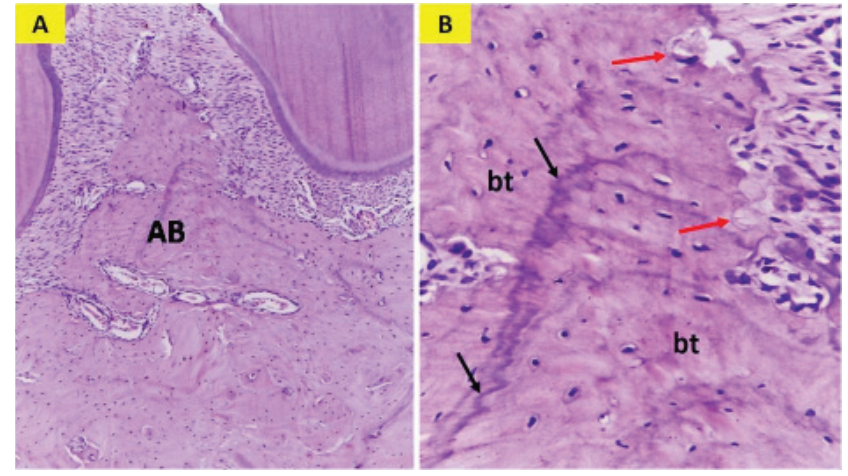

Fig. 2. Photomicrograph of group 2: (A) showing interdental alveolar bone $(A B),(B)$ showing bone trabeculae (bt) containing reversal lines (black arrows) and Howship's lacuna (red arrows) (A: H\&E ×100, B: H\&E ×400)

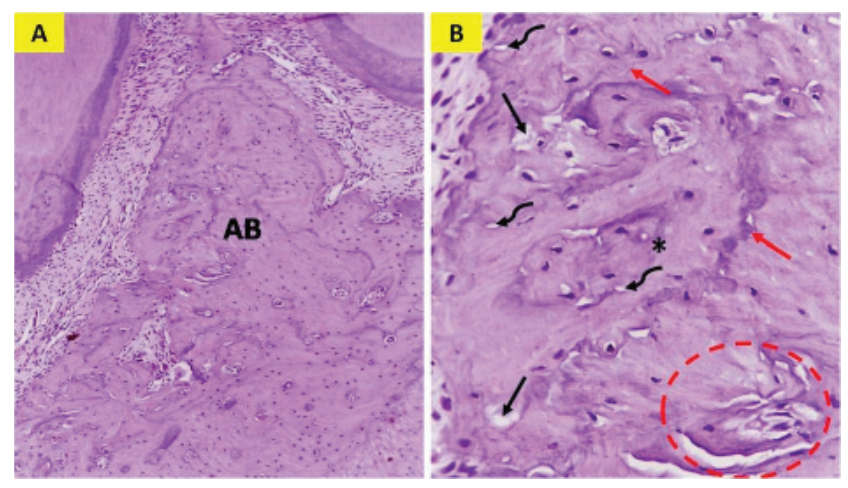

Fig. 3. Photomicrograph of group 3: (A) showing interdental alveolar bone $(A B)$, (B) showing bone trabeculae with multiple vacuoles (black arrows) and degenerated areas (red circle), multiple reversal lines (red arrows) and empty osteocytes lacunae (curved black arrows) (A: H\&E ×100, B: H\&E ×400)

\section{Scanning electron microscopic results}

The SEM images of the control group showed normal smooth alveolar bone architecture (Fig. 4).

SEM examination of group 2 demonstrated alterations of normal bone architecture. The bone surface revealed roughness as well as pore formation. Peeling off of the bone surface with some broken trabeculae was also observed

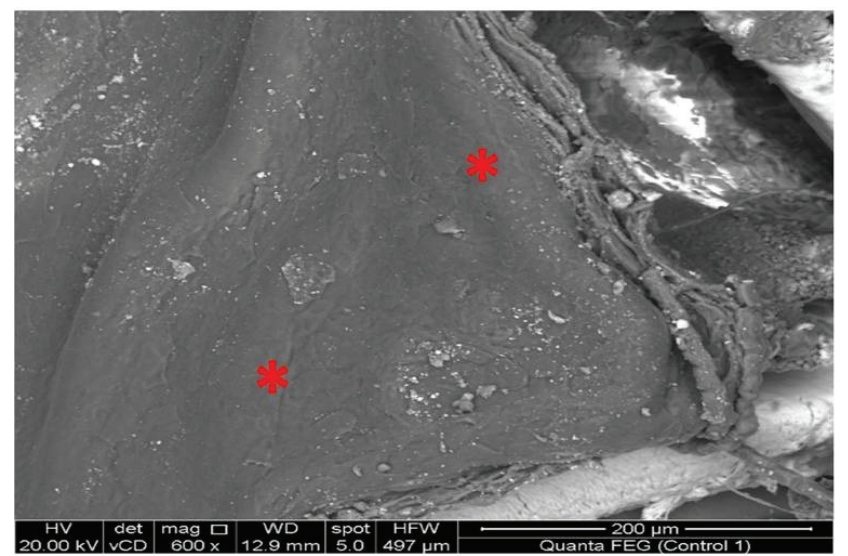

Fig. 4. Scanning electron microscopic photomicrograph of group 1 (control) showing normal smooth alveolar bone architecture (asterisks) (SEM ×600) 
in some areas. Other specimens displayed areas of disintegrated bone architecture with apparent mineralized collagen bundles. These collagen bundles seemed relatively well-organized (Fig. 5).
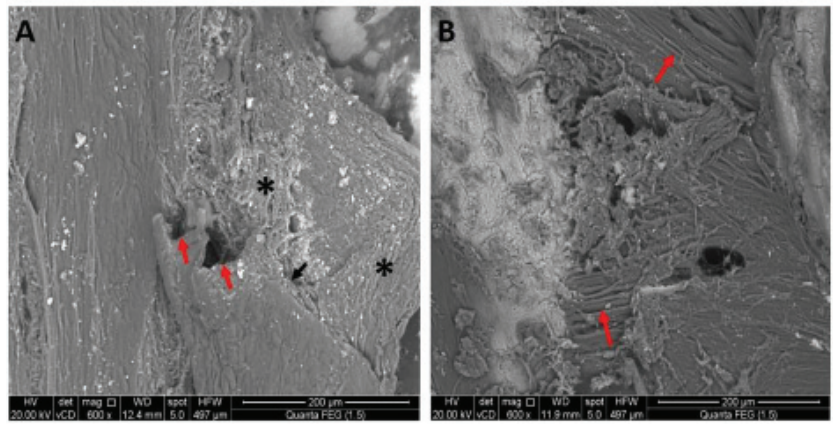

Fig. 5. Scanning electron microscopic photomicrograph of group 2 showing: (A) rough bone architecture (asterisks), peeling off of the bone surface (black arrow) and broken trabeculae (red arrows); (B) areas of disintegrated bone architecture with apparent mineralized collagen bundles (red arrows) (SEM A\&B ×600)

SEM examination of group 3 showed a rough porous eroded bone surface with irregular disorganized bone architecture. The mineralized collagen fibers were seen forming a meshwork. A crack along the bone surface was also observed (Fig. 6).

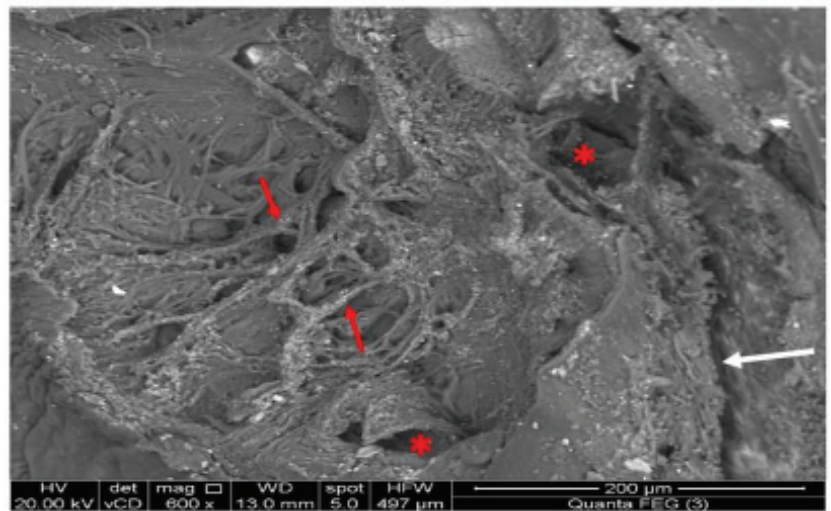

Fig. 6. Scanning electron microscopic photomicrograph of group 3 showing eroded disorganized bone architecture (asterisks), mesh work of mineralized collagen fibers (red arrows) and a crack along the bone surface (white arrow) $($ SEM $\times 600)$

\section{Energy-dispersive X-ray analysis}

The EDX elemental analysis demonstrated the peak of the chemical element - calcium ( $\mathrm{Ca}$ ) concentration - found in the samples in each group. The calcium content in the alveolar bone was evaluated in weight percent (wt\%). EDX quantifies the relative contribution of Ca element to $100 \%$. The mean values \pm SD for total weight percentage of calcium in groups 1, 2 and 3 were calculated (Table 1).
Table 1. Comparison of mean values (mean \pm SD) of calcium weight percentage in the studied groups

\begin{tabular}{|l|c|}
\hline \multicolumn{1}{|c|}{ Studied groups } & Ca weight [\%] \\
\hline Group 1 & $85.79 \pm 0.3725$ \\
Group 2 & $73.468 \pm 0.3182^{\mathrm{a}}$ \\
Group 3 & $69.638 \pm 0.3669^{\mathrm{a}, \mathrm{b}}$ \\
p-value* $^{*}$ & $\leq 0.01$ \\
\hline
\end{tabular}

* ANOVA test; Tukey's post hoc test; a statistically significant with group 1 $(p \leq 0.01) ;{ }^{b}$ statistically significant with group $2(p \leq 0.01)$.

Mean total weight percentage of calcium was highest in group 1 while the lowest was demonstrated in group 3. ANOVA test demonstrated a statistically significant difference among the 3 groups (p-value $\leq 0.01$ ). Post-hoc multiple comparisons between 2 groups revealed that the difference in the mean value was statistically significant between groups 1 and 2, groups 1 and 3, and groups 2 and 3 (p-value $\leq 0.01)$ (Table 1$)$.

\section{Discussion}

Nucleotide inhibitors (NIs) have proved great promise as direct-acting antivirals with broad genotype coverage, lack of preexisting alternatives with reduced susceptibility, a high barrier to resistance, and the ability to produce potent and durable antiviral responses. ${ }^{8}$

The hepatitis $\mathrm{C}$ virus (HCV) infects approx. 3 million individuals in the United States and 180 million worldwide per year. ${ }^{9}$ NIs of HCV RNA synthesis served as alternate substrates and inhibitors of the viral RNA-dependent RNA polymerase HCV nonstructural protein 5B (NS5B). The binding of NIs to the highly conserved NS5B active site resulted in activity that maintained across genotypes and substantial loss of viral fitness upon the infrequent development of resistance mutations. ${ }^{10}$

Sofosbuvir is an oral NI of the HCV NS5B RNA dependent RNA polymerase enzyme, and the mediator demonstrated this activity across all genotypes of HCV. ${ }^{11}$

Although sofosbuvir showed a good secure profile in clinical trials, common side effects have been reported with sofosbuvir treatment in humans. These included headache, insomnia, fatigue, nausea, dizziness, pruritis, upper respiratory tract infections, rash, back pain, grade 1 anemia, and grade 4 lymphopenia. ${ }^{12}$ Thus, we conducted this study to investigate the possible effects of sofosbuvir on alveolar bone histology and surface structure in a rat model.

In the current study, the sofosbuvir dose was calculated so that it was equivalent to the clinically administered dose. The usual total human adult dose of sofosbuvir per day is $400 \mathrm{mg} .{ }^{13}$ According to Shin et al., this dose was equivalent to $8 \mathrm{mg}$ per day for a rat weighing about $200 \mathrm{~g}$. $^{7}$

The alterations in the alveolar process were evaluated in the ongoing research following administration of sofosbuvir for 6 and 12 weeks in groups 2 and 3, respectively. 
These chosen time intervals were based on data from clinical trials reporting that the recommended treatment course duration with sofosbuvir $400 \mathrm{mg}$ once daily is 12 weeks. ${ }^{14}$ However the treatment duration was reported to be guided by on-treatment response and it might be extended to 24 weeks. ${ }^{15}$

The histological results of the present study revealed histopathological changes in the rat alveolar process in both groups 2 and 3 as compared to group 1 . In group 2, the bone trabeculae exhibited irregularly arranged osteocytes. Multiple Howship's lacunae were also clearly seen denoting osteoclastic activity. Moreover, group 3 showed more pronounced changes as compared to group 2, where multiple vacuoles as well as degenerated areas in bone trabeculae were observed, indicating deteriorated bone structure. Empty osteocyte lacunae were observed, which indicate degeneration of osteocytes. Areas of post osteoclastic activity were reflected by the presence of multiple reversal lines in both groups 2 and 3 .

Osteocytes in the current study displayed various changes in both group 2 and 3. These changes suggested bone pathology, as it was reported that osteocytes represent the key responder to various stimuli that regulate bone formation and remodeling as well as one of the key endocrine regulators of bone metabolism. ${ }^{16}$

The scanning electron microscopic results in the research provided good support for the histological findings. In group 2, the alveolar process bone architecture was rough. Moreover, group 3 showed a porous eroded bone surface with irregular disorganized bone architecture.

The relative content of calcium is critical for maintaining mineral homeostasis and bone metabolism. ${ }^{17}$ Ca content was considered a suitable biomarker for the assessment of bone health. ${ }^{18}$ EDX is a sensitive qualitative and semiquantitative technique for the assessment of the mineral content variations in calcified tissue. ${ }^{19}$ The EDX results in the herein study support the SEM findings. The analysis of the $\mathrm{Ca}$ concentration (\%) revealed the highest concentration in the control group while the lowest was found in group 3. Moreover, statistical analysis of the EDX results revealed a statistically significant difference among the studied groups.

It would appear from the reported results that there were obvious alterations in the alveolar bone accompanying sofosbuvir administration. The exact mechanism behind sofosbuvir's effect on the alveolar process is not clear but it may be attributed to the fact that sofosbuvir is a member of the nucleos(t)ide analogues.

Early generations of NIs were reported to cause toxicity. All HCV NIs are ribonucleotide analogues and, therefore, are more likely to attack host RNA polymerases than DNA polymerases. ${ }^{20}$

The mechanisms for toxicity of ribonucleotide analogues were not well characterized but they could be attributed to mitochondrial toxicity. NIs that were incorporated by the mitochondrial RNA polymerase (POLRMT) inhibited mi- tochondrial protein synthesis and showed a corresponding decrease in mitochondrial oxygen consumption in cells. ${ }^{21}$ It has been reported that the use of sofosbuvir for 5 weeks resulted in swollen mitochondria with apparent cristolysis in the rat visual cerebral cortex. ${ }^{22}$

Mitochondrial dysfunction was reported to be the main factor in the formation of excess reactive oxygen species (ROS). The imbalance between these ROS and natural antioxidants created the conditions for oxidative stress. ${ }^{23}$

In bone tissues, recent studies have demonstrated that ROS generation is a key modulator of bone cell function and that oxidative status influences the pathophysiology of mineralized tissues. ROS can adversely affect bone homeostasis so that a proresorptive environment is favored. It was reported that ROS function as a signal mediator in osteoclast differentiation. ${ }^{24,25}$

Cadmium ( $\mathrm{Cd}$ ) has been shown to induce caspaseindependent apoptosis through a mitochondria-ROS pathway. ${ }^{26}$ Zhao et al. investigated the effects of ROS in response to cadmium exposure on osteoblasts in rats. ${ }^{27}$ They clarified that $\mathrm{Cd}$ had direct cytotoxic effect on osteoblasts, which mediated by caspases and mitogen activated protein kinase (MAPK) pathways.

A common dermatologic side effect reported with sofosbuvir was pruritis and rash. ${ }^{28}$ Yonova has reported that pruritis was found to be caused by some chemical substance including histamine. ${ }^{29}$ Another proposed cofactor that might be attributed to the alveolar bone histopathological changes demonstrated in the current study is the effect of histamine. Histamine was reported to be involved during the early phases of strong osteoclast resorption. ${ }^{30}$

\section{Conclusions}

It has been concluded that sofosbuvir induced apparent alterations in rats' alveolar bone. This effect was exaggerated upon a longer period of drug administration. The sofosbuvir-induced alterations might be attributed mainly to mitochondrial toxicity. The effect has been clearly shown histologically and morphologically as well as in bone mineral (calcium) content.

Further clinical and experimental studies are recommended to evaluate the effect of using sofosbuvir on various oral tissues.

\section{References}

1. Gerber L, Welzel TM, Zeuzem S. New therapeutic strategies in HCV polymerase inhibitors. Liver Int. 2013;33:85-92.

2. De Clercq E. A 40 year journey in search of selective antiviral chemotherapy. Annu Rev Pharmacol Toxicol. 2011;51:1-24.

3. Galmarini CM, Mackey JR, Dumontet C. Nucleoside analogues: Mechanisms of drug resistance and reversal strategies. Leukemia. 2001;15:875-890.

4. Lam AM, Espiritu C, Bansal S, et al. Genotype and subtype profiling of PSI-7977 as a nucleotide inhibitor of hepatitis C virus. Antimicrob Agents Chemother. 2012;56:3359-3368. 
5. Johnson AA, Ray AS, Hanes J, et al. Toxicity of antiviral nucleoside analogs and the human mitochondrial DNA polymerase. J Biol Chem. 2001;276:40847-40857.

6. Mc Quaid T, Savini C, Seyedkazemi S. Sofosbuvir, a significant paradigm change in HCV treatment. J Clin Transl Hepatol. 2015;3:27-35.

7. Shin JW, Seol IC, Son CG. Interpretation of animal dose and human equivalent dose for drug development. J Korean Oriental Med. 2010;31:1-7.

8. Sofia MJ. Nucleotide prodrugs for HCV therapy. Antivir Chem Chemother. 2011;22:23-49.

9. Gower E, Estes C, Blach S, Razavi-Shearer K, Razavi H. Global epidemiology and genotype distribution of the hepatitis $C$ virus infection. J Hepatol. 2014;61:45-57.

10. Le Pogam S, Seshaadri A, Kosaka A, et al. Existence of hepatitis $C$ virus NS5B variants naturally resistant to non-nucleoside, but not to nucleoside, polymerase inhibitors among untreated patients. J Antimicrob Chemother. 2008;61:1205-1216.

11. Stedman C. Sofosbuvir, a NS5B polymerase inhibitor in the treatment of hepatitis C: A review of its clinical potential. Therap Adv Gatroenterol. 2014;7:131-140.

12. Bhatia HK, Singh H, Grewal N, Natt NK. Sofosbuvir: A novel treatment option for chronic hepatitis $C$ infection. J Pharmacol Pharmacother. 2014;5:278-284.

13. Gentile I, Borgia F, Buonomo AR, Castaldo G, Borgia G. A novel promising therapeutic option against hepatitis $C$ virus: An oral nucleotide NS5B polymerase inhibitor sofosbuvir. Curr Med Chem. 2013;20:3733-3742.

14. Lawitz E, Mangia A, Wyles D, et al. Sofosbuvir for previously untreated chronic hepatitis C infection. N Engl J Med. 2013;368:1878-1887.

15. Zeuzem S, Dusheiko GM, Salupere R, et al. Sofosbuvir + ribavirin for 12 or 24 weeks for patients with HCV genotype 2 or 3: the VALENCE trial [abstract 1085]. In: Program and abstracts of the 64th Annual Meeting of the American Association for the Study of Liver Diseases. Washington, DC: 2013.

16. Neve A, Corrado A, Cantatore FP. Osteocytes: Central conductors of bone biology in normal and pathological conditions. Acta Physiol (Oxf). 2012;204:317-330.

17. Shapiro R, Heaney RP. Co-dependence of calcium and phosphorus for growth and bone development under conditions of varying deficiency. Bone. 2003;32:532-540.

18. Coats AM, Zioupos P, Aspden RM. Material properties of subchondral bone from patients with osteoporosis or osteoarthritis by microindentation testing and electron probe microanalysis. Calcif Tissue Int. 2003;73:66-71.

19. Kourkoumelis N, Balatsoukas I, Tzaphlidou M. Ca/P concentration ratio at different sites of normal and osteoporotic rabbit bones evaluated by Auger and energy dispersive X-ray spectroscopy. J Biol Phys. 2012;38:279-291.

20. Feng JY, Xu Y, Barauskas O, et al. Role of mitochondrial RNA polymerase in the toxicity of nucleotide inhibitors of hepatitis $C$ virus. Antimicrob Agents Chemother. 2015;60:806-817.

21. Arnold JJ, Sharma SD, Feng JY, et al. Sensitivity of mitochondrial transcription and resistance of RNA polymerase II dependent nuclear transcription to antiviral ribonucleosides. PLoS Pathog. 2012;8:e1003030.

22. Issa NM, El-Sherif NM. Light and electronic histological studies to the effect of Sofosbuvir on the visual cerebral cortex of adult male albino rat. J Am Sci. 2017;13:79-87.

23. Betteridge DJ. What is oxidative stress? Metabolism. 2000;49:3-8.

24. Wauquier F, Leotoing L, Coxam V, Guicheux J, Wittrant Y. Oxidative stress in bone remodeling and disease. Trends $\mathrm{Mol}$ Med. 2009;15:468-477.

25. Moon H, Kim SE, Yun YP, et al. Simvastatin inhibits osteoclast differentiation by scavenging reactive oxygen species. Exp Mol Med. 2011;43:605-612.

26. Shih CM, Ko WC, Wu JS, et al. Mediating of Caspase independent apoptosis by cadmium through the mitochondria-ROS pathway in MRC-5 fibroblasts. J Cell Biochem. 2004;91:384-397.

27. Zhao H, Liu W, Wang Y, et al. Cadmium induces apoptosis in primary rat osteoblasts through Caspase and mitogen-activated protein kinase pathways. J Vet Sci. 2015;16:297-306.
28. Bhatia HK, Singh H, Grewal N, Natt NK. Sofosbuvir: A novel treatment option for chronic hepatitis C infection. J Pharmacol Pharmacother. 2014;5:278-284.

29. Yonova D. Pruritis in certain internal diseases. Hippokratia. 2007;11:67-71.

30. Lesclous P, Schramm F, Gallina S, Baroukh B, Guez D, Saffar JL. Histamine mediates osteoclastic resorption only during the acute phase of bone loss in ovariectomized rats. Exp Physiol. 2006;91:561-570. 